Шалагин С.В. Представление квантового преобразования Фурье на основе дискретной модели квантовомеханической системы

\title{
МАТЕМАТИКА
}

УДК 530.145; 004.383.4

DOI: $10.21779 / 2542-0321-2020-35-4-7-12$

\section{С.В. Шалагин}

\section{Представление квантового преобразования Фурье на основе дискретной модели квантово-механической системы}

ФГБОУ ВПО «Казанский наџиональный исследовательский технический университет им. А.Н. Туполева - КАИ»; Россия, 420111, г. Казань, ул. Карла Маркса, 10; sshalagin@mail.ru

Предложено представление квантового преобразования Фурье (КвПФ) на основе дискретной модели (ДМ) квантово-механической системы, включающей $N=2^{n}$ базисных состояний, которая включает в себя 2(N-1) независимых элементов. Представление основано на однотипных операциях (вентилях): Адамара и с контролируемой фазой. Показана возможность распределенного моделирования КвПФ путем варьирования только N элементов ДМ.

Ключевые слова: квантовое преобразование Фурье, дискретная модель, представление.

\section{Введение}

Исследования в области квантовой обработки информации являются актуальным и перспективным научным направлением [1-8]. Вместе с тем существуют физические барьеры, связанные с реализацией квантово-механических систем (КМС), включающих в себя множество базисных состояний $[4,7]$. В данной связи представляют интерес задачи, связанные с математическим моделированием КМС [6, 9-12].

В [13] предложена дискретная модель (ДМ) КМС, включающая $N$ базисных состояний, которую обозначим как $\mathrm{KMC}(N)$. Состояние $\mathrm{KMC}(N)$ представимо на основе 2(N-1) параметров, а изменение ее состояния - на основе операций умножения над элементами поля Галуа [14], выполняемых параллельно. В [15] показано, что ДМ КМС $(N)$ представима на основе системы однотипных нелинейных полиномиальных функций [16-20], реализуемых на распределенных вычислительных системах с программируемой архитектурой (РВС ПА) [21], элементами которых являются программируемые логические интегральные схемы (ПЛИС) класса FPGA [22].

В работе предложено представление квантового преобразования Фурье (КвПФ) [23], которое основано на определении в рамках ДМ КМС $(N)$, где $N=2^{\mathrm{n}}$, квантового алгоритма, включающего в себя известные квантовые вентили: однокубитный вентиль Адамара и двухкубитный вентиль с контролируемой фазой. Указанное представление позволяет отобразить воздействие КвПФ на $\mathrm{KMC}(N)$, состояние которой описано $2(N-1)$ элементами заданной ДМ путем распределенного варьирования элементов данной ДМ КМС $(N)$ при заданном значении $N=2^{n}$.

Серия 1. Естественные науки. 2020. Том 35. Вып. 4 
Шалагин С.В. Представление квантового преобразования Фурье на основе дискретной модели квантовомеханической системы

\section{Дискретная модель квантово-механической системы с $N$ базисными состояниями}

$\mathrm{KMC}(N)$ общего вида описывается конечномерным гильбертовым (унитарным) пространством $-H,|H|=N<\infty[1,2]$. Состояние $\mathrm{KMC}(N)$ с базисными состояниями $|j\rangle$, $j=\overline{0, N-1}$ описывается как вектор-столбец (кет-вектор) $[2,3]$

$$
|\psi\rangle=\left(\begin{array}{lll}
r_{0} e^{i \varphi_{0}} & \ldots & r_{N-1} e^{i \varphi_{N-1}}
\end{array}\right)^{T},
$$

где $\sum_{j=0}^{N-1} r_{j}^{2}=1$. Система с конечным числом базисных состояний (уровней, измерений $H$ ) представляет интерес с точки зрения квантовой обработки информации. Изменение состояния КМС, представленной в виде (1), описывается квантовым вентилем (КВ) - матрицей $G$ размерности $N \times N$ [4].

ДМ КМС $(N)$ описывается при использовании графовой модели - двоичного (бинарного) дерева $T$ [13]. Число его вершин, являющихся листьями, равно числу базисных состояний $\mathrm{KMC}(N)$, а число его узлов ветвления $(N-1)$ определяет количество параметров, описывающих амплитудные и фазовые составляющие данной модели $2(N-1)$ [15]. Варьирование состояния ДМ КМС $(N)$, описываемое системой (1), задано квантовым вентилем, унитарной матрицей размерности $N \times N$ вида $G=G_{\varphi} G_{A}$, где матрицы $G_{\varphi}$ и $G_{A}$ размерности $N \times N$ определяют варьирование фазовой и амплитудной составляющей ДМ КМС $(N)$ соответственно. В $[13,15]$ сформулированы две теоремы.

Теорема 1. Квантово-механическая система, имеющая $N$ базисных состояний и определенная согласно (1), представима в виде вектора, включающего $2(N-1)$ параметров

$$
\left(\theta_{0}, \ldots, \theta_{N-2}, t_{0}, \ldots, t_{N-2}\right),
$$

где $\left(\theta_{0}, \ldots, \theta_{N-2}\right)$ определяют амплитудные составляющие элементов вектора (1), а $\left(t_{0}, \ldots, t_{N-2}\right)$ - фазовые составляющие (1).

Теорема 2. Операция по варьированию состояния квантово-механической системы, имеющей $N$ базисных состояний и определенной на основе (1), представлена вектором, включающим $2(N-1)$ параметр

$$
\left(\Delta \theta_{0}, \ldots, \Delta \theta_{N-2}, \Delta t_{0}, \ldots, \Delta t_{N-2}\right),
$$

который однозначно определяет квантовый вентиль $G=G_{\varphi} G_{A}$, где $G_{\varphi}$ и $G_{A}$ имеют размерность $N \times N . G_{\varphi}$ задана параметрами $\left(\Delta t_{0}, \ldots, \Delta t_{N-2}\right)$ и описывает варьирование фазовых составляющих, а $G_{A}$ - параметрами $\left(\Delta \theta_{0}, \ldots, \Delta \theta_{N-2}\right)$ и описывает изменение амплитудных составляющих элементов вектора (1).

Пример. Для КМС(4), заданной согласно $(2),\left(\theta_{0}, \ldots, \theta_{2}, t_{0}, \ldots, t_{2}\right)$, элементы кетвектора вида (1) представимы согласно [13] по формулам $r_{0}=\cos \left(\theta_{0}\right) \cos \left(\theta_{1}\right)$, $r_{1}=\cos \left(\theta_{0}\right) \sin \left(\theta_{1}\right), \quad r_{2}=\sin \left(\theta_{0}\right) \cos \left(\theta_{2}\right), \quad r_{3}=\sin \left(\theta_{0}\right) \sin \left(\theta_{2}\right), \varphi_{0}=\tilde{t}, \varphi_{1}=\tilde{t}+t_{1}$, $\varphi_{2}=\tilde{t}+t_{0}, \varphi_{3}=\tilde{t}+t_{0}+t_{2}$. 
Шалагин С.В. Представление квантового преобразования Фурье на основе дискретной модели квантовомеханической системы

\section{Моделирование квантовых вентилей на основе дискретной модели квантово-механической системы}

КвПФ основано на двух однотипных операциях, описываемых оператором Адамара над одним кубитом (или двумя базисными состояниями $\mathrm{KMC}(N)$ ) и вентилем с контролируемой фазой (ВКФ) над двумя кубитами (или четырьмя базисными состояниями $\mathrm{KMC}(N)), N=2^{n}$. Рассмотрим представление указанных операций над ДМ $\mathrm{KMC}(N)$ вида (1).

Оператор Адамара, воздействующий на два базисных состояния $\mathrm{KMC}(N)-|2 j\rangle$ и $|2 j+1\rangle$, представим в виде: $A_{j}=\frac{1}{\sqrt{2}}\left(\begin{array}{cc}1 & 1 \\ 1 & -1\end{array}\right), j=\overline{0, N / 2-1}$. Рассмотрим параметры $\theta_{j+b}$ и $\Delta \theta_{j+b}, j=\overline{0, N / 2-1}, b=N / 2-1$ векторов вида (2) и (3) соответственно. Для представленной модели имеет место

Утверждение 1. Воздействие оператора Адамара $A_{j}$ на базисные состояния $\operatorname{KMC}(N), N=2^{n},|2 j\rangle,|2 j+1\rangle$ описывается элементом $\Delta \theta_{j+b}$ вектора (3), вычисленного на основе элемента $\theta_{j+b}$ вектора (2)

$$
\Delta \theta_{j+b}=\pi / 4-\theta_{j+b},
$$

где $j=\overline{0, N / 2-1}, b=N / 2-1$, и элементом $\Delta t_{j+b}$ вектора (3) $\Delta t_{j+b}=\pi$.

ВКФ, который воздействует на четыре базисных состояния $\mathrm{KMC}(N)-|2 j\rangle,|2 j+1\rangle$, $|2 k\rangle$ и $|2 k+1\rangle,(N / 2-1) \geq k>j \geq 0$, представим в виде матрицы: $S_{j, k}=\left(\begin{array}{cccc}1 & 0 & 0 & 0 \\ 0 & 1 & 0 & 0 \\ 0 & 0 & 1 & 0 \\ 0 & 0 & 0 & D\end{array}\right)$, $D=\exp \left(i \cdot 2^{-(k-j)} \pi\right), 0 \leq j<k \leq(N / 2-1)$. Рассмотрим элементы $t_{j+b}, t_{k+b}, \Delta t_{j+b}$, $\Delta t_{k+b}, j<k, j, k=\overline{0, N / 2-1}, b=N / 2-1$ векторов вида (2) и (3) соответственно. Справедливо

Утверждение 2. Воздействие вентилем с контролируемой фазой $S_{j, k}$ на базисные состояния $\operatorname{KMC}(N), N=2^{n},|2 j\rangle,|2 j+1\rangle,|2 k\rangle$ и $|2 k+1\rangle$ описывается элементом $\Delta t_{k+b}=2^{-(k-j)} \pi$ вектора (3) на элемент $t_{k+b}$ вектора (2), $b=N / 2-1, k=\overline{j+1, N / 2-1}$ при заданном $j=\overline{0, N / 2-2}$.

Реализация КвПФ над КМС $(N), N=2^{n}$ предполагает выполнение квантового алгоритма в виде определенной последовательности воздействий на ее заданные базисные состояния, описываемые квантовыми вентилями вида $A_{j}$ и $S_{j, k}, k=\overline{j+1, N / 2-1}$, $j=\overline{0, N / 2-2}$. Данная последовательность имеет вид [22]: $A_{N / 2-1}, S_{N / 2-2, N / 2-1}, A_{N / 2-2}$, $S_{N / 2-3, N / 2-1}, S_{N / 2-3, N / 2-2}, \ldots, A_{1}, S_{0, N / 2-1}, \ldots, S_{0,1}, A_{0}$. То есть вентили Адамара применяются в обратном порядке к парам базисных состояний $\mathrm{KMC}(N)$ с номерами от $N / 2-1$ до 0. В промежутках между вентилями Адамара $A_{j+1}$ и $A_{j}$ применяются вентили $S_{j, k}$ для $k=\overline{N / 2-1, j+1}$ при заданном $j=\overline{N / 2-2,0}$. Согласно утверждениям 1 и 2 , 
Шалагин С.В. Представление квантового преобразования Фурье на основе дискретной модели квантовомеханической системы

указанные воздействия на $\mathrm{KMC}(N), N=2^{n}$ могут быть представлены в рамках предложенной ДМ $[13,15]$ и вычислены распределенно путем варьирования различных элементов вектора (2) по квантовым вентилям, которые описываются соответствующими элементами вектора (3). Различные вентили Адамара оказывают воздействия $\Delta \theta_{j+b}$, описанные согласно (4), на соответствующие элементы $\theta_{j+b}$ вектора (2), $j=\overline{0, N / 2-1}$, $b=N / 2-1$. Совокупность воздействий, описываемых вентилями Адамара, на элементы $t_{j+b}$ вектора (2), $j=\overline{0, N / 2-1}, b=N / 2-1$, и вентилями с контролируемой фазой $S_{j, k}$, $k=\overline{j+1, N / 2-1}, j=\overline{0, N / 2-2}$, в рамках КвПФ представлена как воздействия вида:

$$
\Delta t_{N / 2-1}=\pi, \Delta t_{v}=\pi\left(1+\sum_{l=1}^{v-b} 2^{-l}\right),
$$

где $v=\overline{N / 2, N-2}, b=N / 2-1$. При этом вентили Адамара и вентили с контролируемой фазой воздействуют на различные параметры независимо друг от друга и порядок их воздействия не имеет значения в рамках ДМ КМС $(N)$. Имеет место

Замечание. При описании КвПФ на основе ДМ КМС $(N), N=2^{n}$ параметры $\theta_{a}$, $t_{a}$ вектора (2) и параметры $\Delta \theta_{a}, \Delta t_{a}$ вектора (3), $a=\overline{0, b-1}, b=N / 2-1$ не задействованы.

На основе вышеизложенного справедливо

Утверждение 3. Воздействие КвПФ на $\mathrm{KMC}(N), N=2^{n}$ представлено как распределенные воздействия, задаваемые согласно (4) - на параметры $\theta_{j+b}, j=\overline{0, N / 2-1}$, и согласно (5) - на параметры $t_{v}, v=\overline{N / 2-1, N-2}, b=N / 2-1$.

Согласно утверждению 3 и замечанию оценим вычислительную сложность моделирования КвПФ КМС $(N), N=2^{n}$ на основе ДМ, состояние которой представлено согласно (2), а варьирование ее состояния - согласно (3). Для представления ДМ КМС $(N)$, $N=2^{n}$ требуется $2(N-1)$ элементов, из которых варьируется только $N: N / 2$ элементов меняют свое значение согласно формуле (4), остальные - согласно формуле (5).

\section{Заключение}

Предложено представление квантового преобразования Фурье при использовании ДМ КМС $(N), N=2^{n}$ путем распределенного варьирования $N$ из $2(N-1)$ элементов указанной ДМ. Вычислены значения величин, на которые производится варьирование элементов ДМ заданной КМС $(N)$. Данное обстоятельство позволяет выполнять моделирование указанного квантового алгоритма при использовании распределенных вычислений. Показано, что количество варьируемых параметров предложенной дискретной модели возрастает линейно с ростом количества базисных состояний $\mathrm{KMC}(N)$.

\section{Литература}

1. Дирак П. Лекции по квантовой механике. - Ижевск: Ижевская республиканская типография, 1998. - 148 с.

2. Холево А.С. Вероятностные и статистические аспекты квантовой теории. - М.: Наука, 1980. - 320 c. 
Шалагин С.В. Представление квантового преобразования Фурье на основе дискретной модели квантовомеханической системы

3. Холево А.С. Введение в квантовую теорию информации. - М.: МЦНМО, 2002.$228 \mathrm{c}$.

4. Валиев К.А., Кокин А.А. Квантовые компьютеры: надежды и реальность. - М.: Ижевск: R \& C Dynamics, 2001. - 351 c.

5. A Quantum Transistor Based on an Atom-Photon Molecule / S.O. Tarasov, N.M. Arslanov, S.A. Moiseev, S.N. Andrianov // Bulletin of the Russian Academy of Sciences: Physics. - 2018. - Yol. 82, № 8. - P. 1042-1046.

6. Аблаев Ф.М. О сложности классических и квантовых моделей вычислений // Математические вопросы кибернетики. - 2004. - Вып. 13. - С. 137-146.

7. Богданов Ю.И., Валиев К.А., Кокин А.А. Квантовые компьютеры: достижения, трудности реализации и перспективы // Микроэлектроника. - 2011. - Т. 40, № 4. C. 243-255.

8. Andrew M. Childs, Wim van Dam. Quantum algorithms for algebraic problems // Rev. Mod. Phys. 82, 1. - Published 15 January 2010. URL: https://journals.aps.org/rmp/abstract/10.1103/RevModPhys.82.1

9. Richter M., Arnold G., Trieu B., Lippert T. Massively Parallel Quantum Computer Simulations: Towards Realistic Systems // John von Neumann Institute for Computing: NIC series. $-2007 .-$ V. 38. - P. 61-68.

10. Богданов Ю.И., Богданова Н.А., Лукичев В.Ф. и др. Вычислительные задачи моделирования элементной базы квантовых компьютеров // Информационные технологии и вычислительные системы. - 2013. - № 3. - С. 3-15.

11. Зуев С.В. Моделирование квантовых вычислений на классическом компьютеpe // Вестник БелГТУ. - 2013. - № 2. - С. 135-139.

12. Statistical Models and Adequacy Validation for Optical Quantum State Tomography with Quadrature Measurements / Y.I. Bogdanov, N.A. Bogdanova, L.V. Belinsky, V.F. Lukichev // Russian Microelectronics. - 2017. - Vol. 46, № 6. - P. 371-378.

13. Шалагин C.В. Дискретная модель квантовой системы обработки информации // Вестник КГТУ им. А.Н. Туполева. - 2007. - № 4. - С. 22-27.

14. Лидл Р., Нидеррайтер Г. Конечные поля: в 2 т. - М.: Мир, 1988.

15. Шалагин C.B. Полиномиальная модель квантовой системы обработки информации // Фундаментальные и прикладные проблемы математики и информатики: материалы XIII Межд. конф., приуроченной к 55-летию факультета математики и компьютерных наук (г. Махачкала, 16-20 сентября 2019 г.). - Махачкала: Изд-во ДГУ, 2019. C. $180-185$.

16. Захаров В.М., Шалагин С.В. Вычисление нелинейных полиномиальных функций на многопроцессорной системе с программируемой архитектурой // Информационные технологии. - 2012. - № 5. - С. 6-11.

17. Захаров В.М., Шалагин С.В. О развитии аппаратных средств статистического моделирования // Развитие вычислительной техники и ее программного обеспечения в России и странах бывшего СССР: история и перспективы: сб. трудов Третьей межд. конф. SoRuCom-2014. - Н. Новгород, 2014. - С. 103-109.

18. Шалагин C.B. Представление нелинейных полиномов над конечным полем распределенной вычислительной системой // Нелинейный мир. - 2009. - № 5. - С. 376379.

19. Шалагин С.В. Сложность вычисления нелинейных полиномиальных функций над полем GF(2^2) на ПЛИC/FPGA // Поиск эффективных решений в процессе создания и реализации научных разработок в российской авиационной и ракетно-косми- 
Шалагин С.В. Представление квантового преобразования Фурье на основе дискретной модели квантовомеханической системы

ческой промышленности: сб. трудов Межд. научно-практ. конф. (г. Казань, 5-8 августа 2014 г.). - Казань, 2014. - С. 661-664.

20. Шалагин C.B. Реализация цифровых устройств в архитектуре ПЛИС/FPGA при использовании распределенных вычислений в полях Галуа: монография. - Казань: КНИТУ-КАИ, 2016. - 228 с.

21. Дордопуло А.И., Каляев И.А., Левин И.И. и др. Высокопроизводительные реконфигурируемые вычислительные системы // Суперкомпьютеры. - 2010. - № 3 (3). C. 44-48.

22. Кузелин М.О., Кнышев Д.А., Зотов В.Ю. Современные семейства ПЛИС фирмы Xilinx: справочное пособие. - М.: Горячая линия - Телеком, 2004. - 440 с.

23. Coppersmith Down. An approximate Fourier transform useful in quantum factoring // IBM Research Report RC19642 «R. Cle». - 1994.

Поступила в редакциию 19 февраля 2020 г.

UDC 530.145; 004.383.4

DOI: $10.21779 / 2542-0321-2020-35-4-7-12$

\section{Representation of the Quantum Fourier Transform Based on a Discrete Model of a Quantum-mechanical System}

\section{S.V. Shalagin}

Kazan National Research Technical University named after A.N. Tupolev-KAI; Russia,420111, Kazan, Karl Marks st., 10; sshalagin@mail.ru

A representation of the quantum Fourier transform (QFT) is proposed based on the discrete model (DM) of a quantum-mechanical system that includes $N=2^{n}$ basic states, involving $2(\mathrm{~N}-1)$ independent elements. The representation is based on the same type of operations (gates): Hadamard and with controlled phase. The possibility of distributed simulation of QFT by varying only $\mathrm{N}$ elements of DM is shown.

Keywords: quantum Fourier transform, discrete model, representation.

Received 19 February 2020 